\title{
Aferesi terapeutica nelle malattie del Sistema Nervoso Centrale
}

\author{
Carlo Tortorella, Vita Direnzo, Pietro Iaffaldano, Elena Luciannatelli, Maria Trojano
}

Dipartimento di Scienze Mediche di Base, Neuroscienze e Organi di Senso, Università degli Studi di Bari, Bari

\begin{abstract}
THERAPEUTICAL APHERESIS IN CENTRAL NERVOUS SYSTEM DISEASES
Abstract. Therapeutical apheresis (TA) has been successfully used in the treatment of exacerbation of demyelinating central nervous system (CNS) diseases that have not responded to first-line treatment with corticosteroids. Nevertheless, these evidences need to be further confirmed according to the American Academy of Neurology guidelines. The main body of data concerns multiple sclerosis and neuromyelitis optica, which respectively involve mainly the T and B-cell CNS immune responses. TA is ineffective in chronic or secondary progressive multiple sclerosis.
\end{abstract}

Key words: Multiple sclerosis, Neromyelitis optica, Therapeutical apheresis

Conflict of interest: None.

Financial support: None.

Ricevuto: 30 Gennaio, 2013; Accettato: 4 Marzo 2013

\section{Introduzione}

Le metodiche di aferesi terapeutica costituiscono un capitolo importante della terapia delle malattie immunomediate del Sistema Nervoso Centrale (SNC) e Periferico (SNP). I dati più consistenti oggi a disposizione riguardano tuttavia essenzialmente la metodica di Plasma Exchange (PLEX), essendo ancora limitate le evidenze relative alle tecniche di Filtrazione a Cascata e Immunoadsorbimento.

È recente la pubblicazione da parte dell' American Academy of Neurology (1) di una sintesi di quelle che sono le evidenze a favore dell'uso del PLEX nelle patologie neurologiche. A differenza di quello che accade in molte malattie del SNP, dove l'uso del PLEX è supportato da evidenze chiare e definite e da studi randomizzati e controllati di Classe I, nelle patologie del SNC la disponibilità di studi controllati randomizzati in doppio cieco è inferiore e spesso si tratta di studi condotti su gruppi eterogenei di pazienti che non consentono di giungere a conclusioni definitive. Va considerato, infatti, che molte delle malattie del SNC che potrebbero giovarsi di aferesi terapeutica sono malattie rare a bassa incidenza, spesso con carattere di cronicità, in cui il PLEX è solo parte dello scenario terapeutico, garantendo perlopiù un effetto a "breve termine". L'associazione precoce con un trattamento immunosoppressivo si rende quasi sempre indispensabile anche per controllare la possibilità di un rebound di produzione di IgG connesso alla rapida eliminazione delle stesse dal circolo.
Il razionale dell'uso dell'aferesi terapeutica nelle malattie immunomediate del SNC e non solo in quelle a prevalente componente $\mathrm{B}$ è la rimozione di autoanticorpi circolanti, ma anche di immunocomplessi, citochine e altri mediatori dell'infiammazione. $\mathrm{E}$, inoltre, dimostrato un effetto disturbante dell'aferesi sul network delle cellule T, dei macrofagi e delle plasmacellule.

Le patologie del SNC per le quali sono disponibili maggiori evidenze di efficacia sono le malattie demielinizzanti, in particolare la Sclerosi Multipla (SM) e la Neuromielite Ottica (NMO) di Devic. Su queste due patologie si concentrerà la discussione del presente articolo.

\section{Aferesi terapeutica nella sclerosi multipla}

La SM è la seconda causa di disabilità neurologica nei giovani. È una patologia autoimmunitaria infiammatoria cronica del SNC a eziopatogenesi multifattoriale (2) ed estremamente eterogenea in termini di decorso clinico, caratteristiche neuroradiologiche delle lesioni, coinvolgimento di geni di suscettibilità e risposta alla terapia. Da un punto di vista patogenetico, in un contesto di suscettibilità genetica, l'attivazione di cellule $\mathrm{T}$ autoreattive e il loro ingresso nel SNC determinano il danno tissutale mediante il rilascio di citochine infiammatorie, la stimolazione di linfociti B e macrofagi e l'attivazione del sistema del complemento.

La forma più comune di SM è caratterizzata dalla comparsa 
acuta/subacuta di deficit neurologici focali che possono poi regredire completamente o parzialmente nell'arco di giorni o settimane (decorso Recidivante-Remittente, RR). Nella maggior parte dei pazienti con SM, dopo circa 15 anni di malattia, il decorso RR lascia il posto a una lenta e continua progressione dei deficit neurologici. Tale fase è nota come Secondariamente Progressiva (SP). In una minoranza di casi, il decorso clinico della SM è caratterizzato da una lenta, graduale e inesorabile progressione dei sintomi neurologici sin dall'esordio (forme Primariamente Progressive).

Da un punto di vista neuropatologico, sono stati recentemente descritti 4 diversi pattern della malattia (3): due pattern (I e II) presentano caratteristiche patologiche simili rispettivamente all'encefalomielite T mediata o a quella T e B mediata e sono caratterizzati da infiltrati infiammatori perivenulari e alti livelli di citochine e il pattern II è caratterizzato anche da depositi di immunocomplessi e frazioni del complemento attivate. Gli altri due pattern (III e IV) sono prevalentemente caratterizzati da una patologia oligodendrocitaria che ricorda più la demielinizzazione indotta da virus o tossine che quella su base autoimmune.

I pattern patologici tendono ad avere una sostanziale omogeneità intra-individuale, nel contesto di lesioni attive del medesimo paziente e condizionano verosimilmente il decorso clinico della malattia (pattern III e IV più frequenti nelle forme progressive) e la risposta alla terapia.

La terapia degli attacchi acuti si basa sull'utilizzo di steroidi ev ad alto dosaggio; numerosi farmaci immunomodulanti/immunosoppressivi sono, invece, approvati come farmaci modificanti il decorso della malattia.

In considerazione della patogenesi della SM e dei meccanismi d'azione dell'aferesi terapeutica, questa metodica può avere un ruolo essenzialmente nella terapia delle riesacerbazioni della SM. Un unico studio classificato come di classe I (4) ha dimostrato che la plasmaferesi è efficace come terapia delle ricadute in aggiunta al bolo steroideo nei pazienti affetti da SMRR. L'endpoint dello studio era rappresentato dal miglioramento della disabilità neurologica a un mese dal trattamento. Un ulteriore studio randomizzato, controllato, in doppio cieco ed effettuato su una popolazione eterogenea di pazienti affetti da patologie infiammatorie acute del SNC (SM, Encefalomielite Acuta Disseminata, Neurite Ottica e Mielite Trasversa) che non avevano risposto alla terapia steroidea ad alte dosi ha dimostrato un beneficio del PLEX a vantaggio del placebo (42\% vs 5.9\%) nei 6 mesi successivi al trattamento (5 sedute di PLEX a giorni alterni) (5). A causa dell'eterogeneità del campione e dei diversi meccanismi eziopatogenetici alla base delle patologie incluse nello studio, questo è stato giudicato di Classe II, con un Livello $\mathrm{C}$ di raccomandazione da parte degli esperti dell'AAN.

Più recentemente, due ulteriori studi $(6,7)$ entrambi retrospettici hanno confermato l'efficacia del PLEX in pazienti con forme gravi fulminanti di demielinizzazione del SNC dopo il fallimento di un trattamento steroideo e hanno evidenziato come fattori predittivi di risposta l'inizio precoce della terapia aferetica rispetto all'esordio dei sintomi (entro due/quattro settimane), la conservazione dei riflessi osteotendinei e la presenza di lesioni con effetto massa o "ring enhancement" alla risonanza magnetica nucleare. Tali lavori evidenziano, inoltre, la possibilità di una risposta "ritardata" alla terapia: circa il $6 \%$ di pazienti rispondeva in un tempo variabile fra 60 e 100 giorni (6), anche se un miglioramento al termine del ciclo aferetico costituiva un fattore prognostico favorevole.

La risposta al trattamento aferetico ha verosimilmente, però, radici più profonde e ancora ulteriori sforzi devono essere effettuati per evidenziare biomarker potenziali di risposta. In un elegante studio di correlazione patologico-clinica Keegan et al. (8) dimostrano una relazione fra risposta al PLEX e le caratteristiche patologiche delle placche di demielinizzazione nel singolo paziente: tutti i pazienti con pattern patologico di tipo II, ma nessuno di quelli con pattern I e III miglioravano dopo PLEX.

Il PLEX si è mostrato inefficace nel trattamento delle forme progressive di SM (4).

Pochi sono i dati pubblicati su metodiche di aferesi selettiva. Recentemente, un lavoro con una casistica limitata di pazienti (9) ha valutato l'efficacia dell'immunoadsorbimento (IA) nel trattamento delle neuriti ottiche refrattarie alla terapia steroidea. Il volume di plasma trattato è stato pari a circa $2.500 \mathrm{~mL}$ e il protocollo seguito era costituito da 5 sedute complessive a giorni alterni. I risultati suggeriscono una buona risposta al trattamento ( $72 \%$ di remissione) e un decremento del recettore dell'interleukina 2 nei pazienti rispondenti al trattamento rispetto ai non rispondenti. Lo stesso studio con un'analisi di proteomica delle proteine adsorbite dimostra, durante il trattamento, oltre a una marcata e attesa rimozione di IgG e complemento, anche la rimozione dal plasma di diverse proteine potenzialmente implicate nella patogenesi della malattia (transtiretina, amiloide P, clusterina, gelsolina, kininogeno-1, MBP, CD5L).

Recenti applicazioni dell'aferesi terapeutica nella SM possono, infine, riguardare l'utilizzo di tale metodica per "rimuovere" il farmaco immunomodulante/soppressivo e ripristinare la funzionalità del sistema immunitario in caso di eventi avversi legati all'impiego di nuove terapie. È stato recentemente approvato per la terapia della SM l'uso di un anticorpo monoclonale (Natalizumab), un farmaco anti-alfa 4 integrina, molecola responsabile dell'adesione dei linfociti $\mathrm{T}$ attivati alla barriera ematoencefalica e del successivo passaggio nel SNC. La possibilità di eventi avversi rari ma severi Natalizumab correlati (p. es., leucoencefalopatia multifocale progressiva, PML) potrebbe richiedere la rapida rimozione del farmaco dal circolo. Studi di cinetica hanno dimostrato, infatti, che il PLEX/IA (5 sedute a giorni alterni) accelera la clearance di Naltalizumab riducendo la sua concentrazione, dopo 7 giorni dall'assunzione, a meno di 1 microg/mL (concentrazione attesa per la desaturazione del recettore delle alfa 4 integrine) alla $15^{\text {a }}$ giornata; la stessa concentrazione sarebbe attesa, in assenza di PLEX, al $97^{\circ}$ giorno dalla sospensione della terapia (10).

Tuttavia, l'ipotesi che il PLEX o l'IA possano accelerare una sindrome da immunoricostituzione (IRIS) rendendosi responsabili di un peggioramento clinico dopo una fase di iniziale beneficio va tenuta in attenta considerazione (11) e ulteriori studi mirati a stabilire eventuali fattori prognostici in grado di predire tale evenienza sono a oggi in corso. 


\section{Aferesi terapeutica nella neuromielite ottica di Devic}

La NMO è una patologia demielinizzante del SNC caratterizzata dall'interessamento preminente del nervo ottico e del midollo spinale. Per anni è stata considerata una variante della SM. Studi clinici più recenti hanno, invece, dimostrato che la NMO è un'entità nosografica a sé che si differenzia dalla SM per caratteristiche cliniche (età più avanzata, decorso più severo), radiologiche (assenza di lesioni encefaliche all'esordio, presenza di estese lesioni midollari con aspetto necrotico), liquorali (presenza di una marcata pleiocitosi e frequente assenza di bande oligoclonali nel liquor cerebrospinale) e patologiche (danno astrocitario più che oligodendrogliale, marcata deposizione di IgG, IgM e complemento tipici delle placche con pattern II della classificazione di Lassmann). La recente identificazione (12) di un autoanticorpo altamente specifico nel siero dei pazienti affetti da NMO (NMO-IgG), che riconosce come antigene l'Acquaporina 4 (AQP-4), una proteina canale per l'acqua espressa a livello dei processi astrocitari perivascolari, supporta tale ipotesi sancendo una differenza anche patogenetica fra le due malattie.

Gli Ac anti-AQP4 hanno, nella NMO, oltre a un ruolo patogenetico, anche un ruolo prognostico. All'esordio della malattia sono predittivi di successive ricadute cliniche; il loro titolo aumenta prima e nel corso della fase di ricaduta, mentre si riduce in fase di remissione e si correla con la gravità clinica dell'attacco (13). La terapia immunosoppressiva, così come l'aferesi terapeutica, determinano una riduzione del titolo anticorpale anti-AQP4, mentre un aumento progressivo del titolo anticorpale nei pazienti che hanno effettuato una terapia con rituximab si associa a un incremento delle cellule CD19 positive e può costituire un parametro per valutare la necessità di un nuovo ciclo terapeutico (13).

L'accumulo della disabilità nella NMO, a differenza di quanto accade nella SM, è strettamente legato al numero e alla gravità delle ricadute cliniche, essendo rare le forme a decorso progressivo di NMO. È, quindi, di primaria importanza trattare le ricadute e instaurare terapie preventive con immunosoppressori. Una caratteristica comune alle ricadute della NMO è, peraltro, la parziale risposta alla terapia steroidea ad alte dosi. La patogenesi preminentemente umorale della malattia e la correlazione ricaduta/titolo anticorpale rende plausibile l'efficacia delle procedure di aferesi terapeutica nelle esacerbazioni non rispondenti o parzialmente rispondenti allo steroide ad alte dosi in pazienti con NMO (14). Tuttavia, la bassa prevalenza della NMO rende indisponibili studi con numerosità sufficiente per poter soddisfare le norme imposte dalla evidence based medicine, che attribuisce a tale terapia nella NMO un livello di evidenza IIb e una raccomandazione di grado C. Gli studi disponibili suggeriscono, tuttavia, l'efficacia dell'aferesi terapeutica sia nelle forme di NMO anti-AQP4 positive che in quelle in cui non è dimostrabile l'Ac sierico (15).

L'ipotesi che l'aferesi terapeutica intermittente possa, infine, essere utile come terapia di mantenimento nei pazienti con NMO non in grado di tollerare la terapia immunosoppressiva è stata suggerita da evidenze di singoli casi e deve essere confermata da successivi studi.

\section{Riassunto}

L'aferesi terapeutica (AT) è stata utilizzata con successo nel trattamento delle ricadute non rispondenti alla terapia steroidea in corso di malattie demielinizzanti. Tuttavia, tali evidenze necessitano tuttora di essere confermate secondo quanto espresso dalle Linee Guida dell'American Academy of Neurology. I dati più sostanziali riguardano le ricadute in corso di Sclerosi Multipla e Neuromielite Ottica. L'AT si è mostrata inefficace nel trattamento della Sclerosi Multipla a decorso progressivo.

Parole chiave: Sclerosi Multipla, Neuromielite Ottica, Aferesi terapeutica

Dichiarazione di conflitto di interessi: Gli Autori dichiarano di non avere conflitto di interessi.

Contributi economici degli autori: Gli Autori dichiarano di non aver ricevuto sponsorizzazioni economiche per la preparazione dell'articolo.

Indirizzo degli Autori:

Dr.ssa Carla Tortorella

Clinica Neurologica "L. Amaducci"

Policlinico Consorziale

Piazza G. Cesare 11

70125 Bari

carla.tortorella@gmail.com

\section{Bibliografia}

1. Cortese I, Chaudhry V, So YT, et al. Evidence-based guideline update: Plasmapheresis in neurologic disorders Report of the Therapeutics and Technology Assessment Subcommittee of the American Academy of Neurology. Neurology 2011; 76: 294-300.

2. Ramagopalan SV, Dobson R, Meier UC, Giovannoni G. Multiple sclerosis: risk factors, prodromes, and potential causal pathways. Lancet Neurol 2010; 9: 727-39.
3. Lassmann H, Brück W, Lucchinetti CF. The immunopathology of multiple sclerosis: an overview. Brain Pathol 2007; 17: 210-8.

4. Weiner HL, Dau PC, Khatri BO, et al. Double-blind study of true vs. sham plasma exchange in patients treated with immunosuppression for acute attacks of multiple sclerosis. Neurology 1989; 39: 1143-9.

5. Weinshenker BG. Therapeutic Plasma Exchange for Acute Inflammatory Demyelinating Syndromes of the Central Nervous 
System. J Clin Apher 1999; 14: 144-8.

6. Llufriu S, Castillo J, Blanco Y. Plasma exchange for acute attacks of CNS demyelination: Predictors of improvement at 6 months. Neurology 2009; 22: 949-53.

7. Magaña SM, Keegan M, Weinshenker BG, et al. Beneficial Plasma Exchange Response in CNS Inflammatory Demyelination. Arch Neurol 2011; 68: 870-8.

8. Keegan M, König F, McClelland R, et al. Relation between humoral pathological changes in multiple sclerosis and response to therapeutic plasma exchange. Lancet 2005; 366: 579-82.

9. Koziolek M, Tampe D, Bähr M, et al. Immunoadsorption therapy in patients with multiple sclerosis with steroid-refractory optical neuritis. J Neuroinflammation 2012; 9: 1-10.

10. Khatri BO, Man S, Giovannoni G, et al. Effect of plasma exchange in accelerating natalizumab clearance and restoring leucocyte function. Neurology 2009; 72: 402-9.

11. Tan IL, McArthur JC, Clifford DB, et al. Immune reconstitution inflammatory syndrome in natalizumab-associated PML. Neurology 2011; 77: 1061-7.

12. Lennon VA, Wingerchuk DM, Kryzer TJ, et al. A serum autoantibody marker of neuromyelitis optica: distinction from multiple sclerosis. Lancet 2004; 364 (9451): 2106-12.

13. Jarius S, Aboul-Enein F, Waters $\mathrm{P}$, et al. Antibody to aquaporin-4 in the long-term course of neuromyelitis optica. Brain 2008; 131: 3072-80.

14. Watanabe S, Nakashima I, Misu T, et al. Therapeutic efficacy of plasma exchange in NMO-IgG-positive patients with neuromyelitis optica. Mult Scler 2007; 13 (1): 128-32.

15. Bonnan M, Cabre P. Plasma exchange in severe attacks of neuromyelitis optica. Mult Scler Int 2012; 2012: 1-9. 